\title{
Global Public Health Guidelines on Physical Activity and Sedentary Behavior for People Living With Chronic Conditions: A Call to Action
}

\author{
Paddy C. Dempsey, Christine M. Friedenreich, Michael F. Leitzmann, Matthew P. Buman, \\ Estelle Lambert, Juana Willumsen, and Fiona Bull
}

\begin{abstract}
Background: In 2020, the World Health Organization (WHO) released global guidelines on physical activity (PA) and sedentary behavior, for the first time providing population-based recommendations for people living with selected chronic conditions. This article briefly presents the guidelines, related processes and evidence, and, importantly, considers how they may be used to support research, practice, and policy. Methods: A brief overview of the scope, agreed methods, selected chronic conditions (adults living with cancer, hypertension, type 2 diabetes, and human immunodeficiency virus), and appraisal of systematic review evidence on PA/sedentary behavior is provided. Methods were consistent with World Health Organization protocols for developing guidelines. Results: Moderate to high certainty evidence (varying by chronic condition and outcome examined) supported that PA can reduce the risk of disease progression or premature mortality and improve physical function and quality of life in adults living with chronic conditions. Direct evidence on sedentary behavior was lacking; however, evidence extrapolated from adult populations was considered applicable, safe, and likely beneficial (low certainty due to indirectness). Conclusions: Clinical and public health professionals and policy makers should promote the World Health Organization 2020 global guidelines and develop and implement services and programs to increase PA and limit sedentary behavior in adults living with chronic conditions.
\end{abstract}

Keywords: exercise, clinical medicine, global health, chronic disease, cardiovascular, type 2 diabetes, cancer, hypertension, HIV, AIDS, health promotion, noncommunicable disease

Chronic conditions-including noncommunicable diseases such as cardiovascular disease (CVD), hypertension, cancer, and type 2 diabetes (T2D) and some communicable diseases such as human immunodeficiency virus (HIV) — are diseases of long duration, slow progression, and generally requiring ongoing management over periods of years to decades. Chronic conditions are leading causes of morbidity and mortality globally, contributing substantially toward total public health burden, including some $73.4 \%$ (41 million) of all deaths in 2017. ${ }^{1,2}$ The growing burden of chronic disease is affecting all countries, but disproportionately impacts the poorest and most vulnerable, including low- and middle-income countries undergoing demographic, epidemiological, economic, social, and technological transitions. Indeed, chronic conditions in these settings contribute to large financial and social burdens through lost productivity and health care costs,

\footnotetext{
Dempsey is with the MRC Epidemiology Unit, Institute of Metabolic Science, University of Cambridge, Cambridge Biomedical Campus, Cambridge, United Kingdom; the Baker Heart and Diabetes Institute, Melbourne, VIC, Australia; and the Diabetes Research Centre, University of Leicester, Leicester General Hospital, Leicester, United Kingdom. Friedenreich is with the Department of Cancer Epidemiology and Prevention Research, CancerControl Alberta, Alberta Health Services, Calgary, Canada; and the Departments of Oncology and Community Health Sciences, Cumming School of Medicine, University of Calgary, Calgary, Canada. Leitzmann is with the Department of Epidemiology and Preventive Medicine, University of Regensburg, Germany. Buman is with the College of Health Solutions, Arizona State University, Phoenix, AZ, USA. Lambert is with the Research Centre for Health through Physical Activity, Lifestyle and Sport, Faculty of Health Sciences, University of Cape Town, Cape Town, South Africa. Willumsen and Bull are with the Physical Activity Unit, Department of Health Promotion, World Health Organization, Geneva, Switzerland. Dempsey (paddy.dempsey@ mrc-epid.cam.ac.uk) is corresponding author.
}

posing substantial barriers to sustainable development. ${ }^{3-5}$ Furthermore, with population aging and the high prevalence of physical inactivity and obesity, ${ }^{6,7}$ the number of people with 2 or more chronic conditions (ie, multimorbidity) is predicted to rise rapidly. ${ }^{8,9}$ Aside from already contributing significantly to increases in functional decline, disability, and health care costs, these factors combined represent a formidable set of clinical, economic, and public health challenges..$^{4,10}$

Regular physical activity (PA), or moderate-to vigorousintensity PA (MVPA), is a recognized cost-effective intervention for public health and is associated with an ever-widening constellation of health, ${ }^{11}$ economic, ${ }^{10,12}$ and other co-benefits, ${ }^{13}$ playing an important role in the prevention and management of many major chronic conditions. ${ }^{5,14-16}$ However, people living with chronic conditions are generally less physically active and more sedentary ${ }^{17,18}$ and may, along with health care professionals, be more wary about the potential benefits, risks, or adverse health impacts of these behaviors.

To date, most PA recommendations for adults living with chronic conditions have been limited to clinical practice guidelines, ${ }^{19-22}$ which are typically more focused on immediate clinical care and therapeutic services and are used in consultation with individual patients. Public health recommendations aim to provide safe and effective guidelines at the population level, focusing on disease prevention/management and health promotion over longer term timescales. Thus, information from both clinical practice guidance and public health recommendations is complementary, and can provide patients, health care professionals, and policymakers with clearer advice on the role of PA and sedentary behavior based on considerations around potential efficacy, feasibility as well as risks/safety, and cost-effectiveness and support for broader community and health care services and programs. 
In 2018, the World Health Organization (WHO) was requested to update the 2010 recommendations based on the latest available evidence as part of global efforts to support countries to implement recommendations set out in the WHO Global Action Plan on Physical Activity 2018-2030 (GAPPA) and achieve a 15\% reduction in inactivity by $2030 .{ }^{13}$ The WHO established an international group of public health scientists and practitioners to serve on the Guidelines Development Group (GDG) ${ }^{23}$ and, for the first time, included within its scope the development of recommendations on PA and sedentary behavior for adults living with chronic conditions - specifically those living with cancer (cancer survivors), hypertension, T2D, and HIV. This expanded scope fills important global health policy gaps. The purpose of the present article is to briefly summarize the development process and highlight the scientific evidence that underpins the new global population health guidelines. Importantly, it also considers how these new guidelines may be used to both inform and improve clinical and population health practice, policy, and future research endeavors.

\section{Methods}

The WHO convened the first meeting of the GDG in July 2019 to review and finalize the scope and agree on the methods for this work following WHO guidelines development protocols. ${ }^{24}$ The health benefits of PA in secondary prevention of coronary heart disease have been previously established based on a substantial evidence base and were, therefore, not examined. ${ }^{25,26}$ The evidence base around PA and health outcomes in those living with disabilities are detailed in a separate article. ${ }^{27}$ The rationale for the selected chronic conditions and the methods for identifying and grading the most up-to-date evidence to inform the updated WHO Guidelines are described in detail elsewhere. ${ }^{23,28}$

Briefly, the GDG reviewed and finalized the scope of the guidelines, selected the chronic conditions to be examined, and decided on the set of Population, Intervention/Exposure, Comparison, Outcome questions (Table 1). Evidence to support multicomponent PA (ie, emphasizing functional balance and strength training to enhance functional capacity and prevent falls) for older adults living with chronic conditions was largely extrapolated from older adult evidence reviews, whereas the primary evidence base for assessing the associations between sedentary behavior and health outcomes in all adults with chronic conditions was the scientific literature collated and reviewed for adult populations. ${ }^{28,29}$ Review of these findings included assessing if there was evidence that the outcomes would be any different, would not apply to, or would be contraindicated for adults and older adults living with chronic conditions.

The evidence reviews, and resultant guidelines, are primarily focused on the preventive rather than therapeutic effects of either PA or MVPA. That is, prevention of an additional chronic condition (ie, a secondary cancer, T2D, or CVD mortality) or prevention (ie, preventing a chronic condition from worsening over time as assessed by indicators of disease progression). However, some preventive effects are inevitably overlapping with therapeutic effects for some conditions. For example, reducing $\mathrm{HbA}_{1 \mathrm{c}}$ is a preventive risk factor for disease progression, but is also considered a clinical or therapeutic target for T2D management.

Literature searches were undertaken to update the most recent and relevant systematic reviews, which were identified to be the comprehensive syntheses of evidence undertaken and reported by the Physical Activity Guidelines Advisory Committee in $2018^{14}$ for cancer, hypertension, and T2D. The credibility of the new systematic reviews was rated using the Assessment of Multiple Systematic
Reviews 2 instrument ${ }^{30}$ by external reviewers who also used the Grading of Recommendations Assessment, Development and Evaluation method to rate the certainty (very low to high) of the evidence for each Population, Intervention/Exposure, Comparison, Outcome (see Supplementary Material [available online]). ${ }^{31,32}$ A separate and new umbrella review for HIV was also commissioned by the WHO using similar methods.

The GDG considered the entire body of evidence, including evidence reported in the Physical Activity Guidelines Advisory Committee report and new reviews identified as part of the updated searches, to make recommendations on health outcomes and PA and sedentary behavior for adults living with chronic conditionsspecifically hypertension, T2D, cancer, and HIV. Other considerations such as values, preferences, and risks and identified evidence gaps were also appraised. ${ }^{28}$

\section{Results}

The updated systematic reviews for cancer, hypertension, and T2D identified 22 new reviews published from 2016 to August 2019. ${ }^{28}$ Of these, 16 reviews met inclusion criteria $(n=6$ were rated as having critically low credibility and not included in final evidence profiles) and examined the association between PA and the predefined health outcomes among adults living with cancer $(n=1)$, hypertension $(n=2)$, and T2D $(n=13)$. None of the new reviews included evidence published in 2019, and very few included studies published in 2017 or 2018. For HIV, the WHOcommissioned umbrella review identified 24 reviews published between 2002 and 2018 of which 19 met inclusion criteria and 15 were of moderate to high quality.

Table 2 provides a summary of the level of evidence for each chronic condition and health outcome examined. For a detailed summary of the meta-analyses and systematic reviews that contributed new evidence to inform recommendations, see the WHO report ${ }^{28}$ and the Web Annex Evidence Profiles (Tables E.1.4.a-d). ${ }^{28}$ An overview of the key findings is provided next.

\section{Cancer Survivors (Web Annex, Table E.1.4.a)}

A new review ${ }^{33}$ on the role of PA in cancer survival, including 136 observational studies and randomized controlled trials (RCTs), provided evidence that both prediagnosis and postdiagnosis PA reduced the risk of all-cause and cancer-specific mortality for survivors of breast, colorectal, and prostate cancers. Of particular relevance for cancer survivors were the strong risk reductions with postdiagnosis activity. This new evidence was consistent with previous findings, ${ }^{14}$ and it was concluded that, overall, there is moderate-certainty evidence that PA is associated with lower risk of all-cause and cancer-specific mortality among cancer survivors (particularly for breast and colorectal cancer survivors), but insufficient evidence to determine if PA was associated with cancer recurrence or second primary cancer. There was also evidence to support a dose-response relation between PA and survival after breast and colorectal cancers but insufficient evidence regarding this dose-response relation for other cancer sites.

\section{Hypertension (Web Annex, Table E.1.4.b)}

No new reviews beyond those already identified in the Physical Activity Guidelines Advisory Committee report were identified for the association between PA and the risk of comorbid conditions among people living with hypertension. Two new small moderate- to 
Table 1 Scope and PI/ECO Questions Related to Physical Activity and Health Outcomes in Adults Living With Cancer, Hypertension, T2D, and HIV

Guiding questions

What is the association between physical activity and health-related outcomes?

a. Is there a dose response association (volume, duration, frequency, intensity)?

b. Does the association vary by type or domain of PA?

\section{[P]opulation}

People living with Cancer

People living with Hypertension

People living with T2D

People living with HIV

\section{[I]ntervention/[E]xposure}

Greater volume, duration, frequency, or intensity of physical activity

\section{[C]omparison}

No physical activity or lesser volume, duration, frequency, or intensity of physical activity

[O]utcomes (critical)

Cancer (survivors)

All-cause mortality

Cancer-specific mortality

Risk of cancer recurrence or second primary cancer

Hypertension

Risk of co-morbid conditions

Physical function

Health-related quality of life

Disease progression $^{\mathrm{a}}$

T2D

Risk of co-morbid conditions

Physical function

Health-related quality of life

Disease progression $^{\mathrm{b}}$

HIV

Physical function

Health-related quality of life

Mental health (symptoms of depression and anxiety)

Disease progression $^{\mathrm{c}}$

Abbreviations: BMI, body mass index; CVD, cardiovascular disease; T2D, type 2 diabetes; HIV, human immunodeficiency virus; WHO, World Health Organization.

${ }^{\mathrm{a}}$ Defined by: (1) blood pressure response to physical activity (indicator of [risk of] CVD progression) and (2) CVD mortality (indicator of longstanding hypertension). ' ${ }^{\mathrm{b}}$ Defined by: (1) indicators of actual progression/complications (retinopathy, peripheral neuropathy, nephropathy, and diabetes-related foot infections/ foot ulcers) and (2) indicators of risk of progression ( $\mathrm{HbA}_{1 \mathrm{c}}$, blood pressure, $\mathrm{BMI}$, and blood lipids). Note: For disease progression in T2D, only measures of glycemic control were included in the assessment of updated evidence requested by WHO due to the prior existence of high-quality evidence for these outcomes and resource constraints. ${ }^{\mathrm{c}}$ Defined by: (1) cardiometabolic risk indicators (blood lipids, blood glucose, and body composition) and (2) adverse effects on viral load or CD4+ count.

high-certainty reviews indicated that MVPA improves cardiopulmonary fitness as assessed by cardiorespiratory fitness (high-intensity interval training vs moderate aerobic activity; 10 RCTs, $n=245)$ and health-related quality of life (1 RCT, $\mathrm{n}=103)$. Both these reviews also assessed the risk of disease progression, and reported MVPA was associated with improvements in blood pressure among the exercise groups compared with controls (systolic blood pressure mean difference $=-12.26 \mathrm{~mm} \mathrm{Hg}[95 \%$ confidence interval, -15.17 to -9.34]; diastolic blood pressure mean difference $=-6.12 \mathrm{~mm} \mathrm{Hg}$ [ $95 \%$ confidence interval, -7.76 to -4.48$]$ ]. ${ }^{34,35}$ No new reviews were identified in the update on the dose-response relationship between PA and cardiovascular disease mortality; however, existing evidence ${ }^{36-38}$ supported an inverse dose-response relationship between PA and cardiovascular disease mortality.

\section{T2D (Web Annex, Table E.1.4.c)}

No new reviews were identified in updated searches to show an inverse association between PA and risk of cardiovascular mortality in people with T2D. However, data ${ }^{14}$ from 2 meta-analyses and 
Table 2 Summary of Relationships and Level of Evidence for PA With Each Predefined Health Outcome in Adults Living With Cancer, Hypertension, T2D, and HIV

\begin{tabular}{|c|c|c|c|}
\hline Chronic condition & $\begin{array}{l}\text { Evidence for } \\
\text { association }\end{array}$ & Evidence for dose-response & Evidence for type or domain of PA \\
\hline \multicolumn{4}{|l|}{ Cancer (survivors) } \\
\hline All-cause mortality & $\begin{array}{l}\text { Moderate (high for breast, } \\
\text { colorectal) }\end{array}$ & Moderate $^{\mathrm{b}}$ & Insufficient/low \\
\hline $\begin{array}{l}\text { Cancer-specific } \\
\text { mortality }\end{array}$ & $\begin{array}{l}\text { Moderate (high for breast, } \\
\text { colorectal) }\end{array}$ & Moderate $^{\mathrm{b}}$ & Insufficient/low \\
\hline Cancer recurrence & Insufficient & Insufficient & Insufficient \\
\hline \multicolumn{4}{|l|}{ Hypertension } \\
\hline $\begin{array}{l}\text { Risk of co-morbid } \\
\text { conditions }\end{array}$ & Insufficient & Insufficient & Insufficient \\
\hline Physical function & Moderate & Insufficient & Insufficient \\
\hline $\begin{array}{l}\text { Health-related quality } \\
\text { of life }\end{array}$ & Moderate & Insufficient & Insufficient \\
\hline Disease progression & $\begin{array}{l}\text { High (blood pressure, } \\
\text { CVD mortality) }\end{array}$ & Moderate (CVD mortality) & $\begin{array}{l}\text { Low to moderate (effects on BP do not } \\
\text { appear to vary by type/mode of PA, but } \\
\text { evidence more robust for aerobic, } \\
\text { dynamic resistance, or combined PA) }{ }^{b}\end{array}$ \\
\hline \multicolumn{4}{|l|}{$\mathrm{T} 2 \mathrm{D}$} \\
\hline $\begin{array}{l}\text { Risk of co-morbid } \\
\text { conditions }\end{array}$ & High (CVD mortality) ${ }^{\mathrm{b}}$ & Moderate (CVD mortality) ${ }^{\mathrm{b}}$ & Insufficient \\
\hline Physical function & Low & Insufficient & Insufficient \\
\hline $\begin{array}{l}\text { Health-related quality } \\
\text { of life }\end{array}$ & Low & Insufficient & Insufficient \\
\hline Disease progression $^{\mathrm{a}}$ & $\begin{array}{l}\text { Moderate to high }\left(\mathrm{HbA}_{1 \mathrm{c}} \text {, }\right. \\
\text { blood pressure, } \mathrm{BMI}, \\
\text { lipids) }\end{array}$ & $\begin{array}{l}\text { Moderate for volume of aerobic PA (blood } \\
\left.\text { pressure, } \mathrm{HbA}_{1 \mathrm{c}}\right)^{\mathrm{b}}\end{array}$ & $\begin{array}{l}\text { Moderate to high for aerobic, muscle- } \\
\text { strengthening, and both }\end{array}$ \\
\hline \multicolumn{4}{|l|}{ HIV } \\
\hline Physical function & $\begin{array}{l}\text { Moderate }\left(\mathrm{VO}_{2} \mathrm{max} \text { or }\right. \\
\text { exercise tolerance, muscle } \\
\text { strength })\end{array}$ & Insufficient & $\begin{array}{l}\text { Moderate to high for aerobic, muscle- } \\
\text { strengthening, and both }\end{array}$ \\
\hline $\begin{array}{l}\text { Health-related quality } \\
\text { of life }\end{array}$ & Moderate & Insufficient & $\begin{array}{l}\text { Moderate for aerobic and concurrent } \\
\text { aerobic and muscle strengthening } \\
\text { (insufficient for muscle strengthening } \\
\text { alone) }\end{array}$ \\
\hline Mental health & $\begin{array}{l}\text { Moderate (depression- } \\
\text { dejection scale) }\end{array}$ & Insufficient & $\begin{array}{l}\text { Moderate for aerobic (insufficient for } \\
\text { muscle strengthening alone) }\end{array}$ \\
\hline Disease progression & $\begin{array}{l}\text { - Moderate (BF\%, LBM; } \\
\text { no significant change in } \\
\text { viral load or CD4+ count) } \\
\text { - Low (blood lipid profiles, } \\
\text { insulin resistance, fasting } \\
\text { glucose concentrations, or } \\
\text { blood pressure) }\end{array}$ & Insufficient & $\begin{array}{l}\text { Moderate to high for aerobic, muscle- } \\
\text { strengthening, and both (BF\%, LBM; no } \\
\text { significant change in viral load or CD4+ } \\
\text { count) }\end{array}$ \\
\hline
\end{tabular}

Abbreviations: BMI, body mass index; BF\%, percentage body fat; CVD, cardiovascular disease; LBM, lean body mass; PA, physical activity; PAGAC, Physical Activity Guidelines Advisory Committee; $\mathrm{VO}_{2} \mathrm{max}$, cardiorespiratory fitness; T2D, type 2 diabetes; HIV, human immunodeficiency virus.

Note: See the WHO report ${ }^{28}$ for details on the framework to rate the quality/certainty of the evidence and the full evidence profiles.

${ }^{\mathrm{a}}$ Insufficient evidence for retinopathy, peripheral neuropathy, nephropathy, and diabetes-related foot infections/foot ulcers outcomes. ${ }^{\mathrm{b}}$ Level of evidence rating based on evidence from PAGAC reviews. ${ }^{14}$

1 pooled analysis provided substantial evidence of an inverse association between volume of PA and risk of cardiovascular mortality, with evidence for an inverse curvilinear dose-response relationship with PA volume. ${ }^{39-41}$ Moderate- and high-certainty RCT evidence showed that aerobic activity, muscle-strengthening activity, and aerobic plus muscle-strengthening activity are associated with improvements in $\mathrm{HbA}_{1 \mathrm{c}}$, blood pressure, body mass index, and lipids among adults with T2D. ${ }^{14,42,43}$ Although the updated searches identified 3 new low-certainty reviews, it was concluded that there was insufficient evidence on the association between PA and quality of life or physical function.

\section{HIV (Web Annex, Table E.1.4.d)}

Most of the evidence in people living with HIV was based on clinical studies or RCTs comparing structured aerobic or multimodal exercise interventions to no exercise. Moderate-certainty evidence (24 RCTs, $\mathrm{N}=936$ ) showed that MVPA improves health-related quality of life 
(>10-point change), particularly for general health and physical functioning. There was also moderate-certainty evidence to support improvements in body composition and functional capacity, with significant effects on body fat percentage $(\sim 1.12 \%)$, lean body mass $(\sim 1.75 \mathrm{~kg})$, cardiorespiratory fitness, and muscle strength, but no statistically significant effects on body mass index or waist circumference. Moderate-certainty evidence supported improvements in symptoms of depression and anxiety, with 1 meta-analysis showing a 7.68-point improvement in the depression-dejection subscale of the Profile of Mood States Scale. ${ }^{44}$ Evidence on cardiometabolic risk biomarkers (blood lipid profiles, insulin resistance, fasting glucose concentrations, or blood pressure) was inconsistent. Finally, moderate-certainty evidence shows that regular MVPA (aerobic or combined with resistance training) did not result in any significant change in viral load or CD4+ count, suggesting that HIV will not be adversely affected by MVPA.

\section{Type of PA and Multicomponent PA}

There was moderate-high certainty evidence to support different types or modes of PA (ie, mostly focused on specific or combined aerobic or muscle-strengthening modalities) for adults living with T2D, hypertension, and HIV for some of the specific outcomes (see earlier and Web Annex Tables E.1.4. $a-d)$. However, published evidence for cancer survivors and mortality outcomes was lacking, and the level of certainty was, therefore, downgraded for this chronic condition (Tables 2 and 3). ${ }^{14,28}$ The evidence reviewed on multicomponent physical activity for older adults (see Web Annex Tables C.1.a-e) was considered and viewed as applicable (moderate-certainty evidence; assuming no contraindications) and extrapolated to inform recommendations on multicomponent PA for older adults living with chronic conditions for a common set of critical health outcomes (Table 3). ${ }^{14,28}$ The extrapolation of this evidence was supported largely by the assessment that these studies in older adults very likely included people with 1 or more chronic conditions, and no reason was identified for why the evidence would not still apply.

\section{Sedentary Behavior}

There was a notable paucity of direct evidence on sedentary behavior and health outcomes in adults living with chronic conditions. ${ }^{28,29}$ The evidence reviewed on sedentary behaviors in the general adult population, including the benefit of undertaking higher amounts of MVPA to help counteract the potential risks of high levels of sedentary behavior, ${ }^{29}$ was, therefore, considered

\section{Table 3 Key Guidelines for Adults and Older Adults Living With Cancer, Hypertension, T2D, and HIV}

\begin{tabular}{l}
\hline Physical activity \\
\hline It is recommended that: \\
- All adults with chronic conditions should undertake regular PA. ${ }^{\text {a }}$ \\
- Adults with chronic conditions should do at least $150-300$ min of moderate- \\
intensity aerobic PA, or do at least $75-150$ minutes of vigorous-intensity \\
aerobic PA, or an equivalent combination of moderate- and vigorous- \\
intensity activity throughout the week for substantial health benefits. \\
- Adults with chronic conditions should also do muscle-strengthening \\
activities at moderate or greater intensity that involve all major muscle \\
groups on 2 or more days a week, as these provide additional health \\
benefits. \\
- As part of their weekly PA, older adults with chronic conditions should do \\
varied multicomponent PA that emphasizes functional balance and strength \\
training at moderate or greater intensity on 3 or more days a week to enhance \\
functional capacity and prevent falls. \\
- When not contraindicated, adults with chronic conditions may increase \\
moderate-intensity aerobic PA to more than 300 min, or do more than \\
150 min of vigorous-intensity aerobic PA, or an equivalent combination of \\
moderate- and vigorous-intensity activity throughout the week for additional \\
health benefits. ${ }^{\text {c }}$
\end{tabular}

\section{Sedentary behavior ${ }^{\mathrm{d}}$}

It is recommended that:

- Adults with chronic conditions should limit the amount of time spent being sedentary. Replacing sedentary time with PA of any intensity (including light intensity) has health benefits.

- To help reduce the detrimental effects of high levels of sedentary behavior on health, adults with chronic conditions should aim to do more than the recommended levels of moderate to vigorous PA.

Additional explanatory and practical notes:

- All adults with chronic conditions should try to meet these recommendations where possible, as able, and without contraindication.

- Good practice statement: When not able to meet the above recommendations, adults with chronic conditions should aim to engage in PA according to their abilities. They should start with small amounts of PA and gradually increase frequency, intensity, and duration over time.

- Adults with chronic conditions may wish to consult with a PA specialist or health care professional for advice on the types and amounts of activity appropriate for their individual needs, abilities, functional limitations/complications, medications, and overall treatment plan.

- Preexercise medical clearance is generally unnecessary for individuals without contraindications prior to beginning light- or moderate-intensity PA not exceeding the demands of brisk walking or everyday living.

- Those undergoing acute treatment (eg, chemotherapy) or not yet stabilized on their chronic medication should seek advice from their health care provider who may also refer to clinical guidelines relevant to each chronic condition.

Abbreviations: T2D, type 2 diabetes; HIV, human immunodeficiency virus; PA, physical activity.

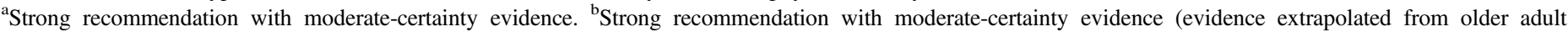

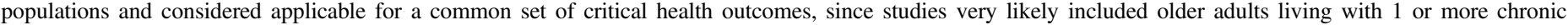

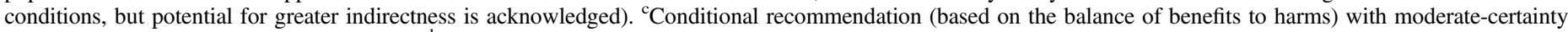

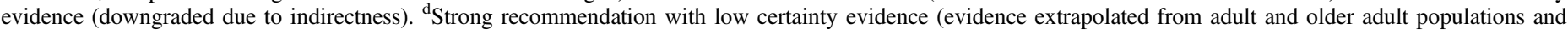

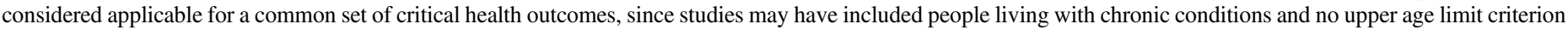
was imposed; evidence level downgraded to very low certainty for adults living with HIV due to indirectness). 
and viewed as applicable (assuming no contraindications) and extrapolated to inform new WHO recommendations on sedentary behavior for adults living with chronic conditions for a common set of critical health outcomes (low-certainty evidence; Table 3). The extrapolation of evidence was supported largely by the assessment that the majority of studies: (1) imposed no upper age limit criterion, (2) included adults over the age of 65 years, and (3) may have included adults with chronic conditions-such as those living with cancer, T2D, or hypertension. For HIV, no reason was identified for why the evidence on the health impacts of sedentary behaviors would not also still apply, but due to the indirectness of the evidence to develop these recommendations, the level of certainty was downgraded (Table 3).

The final guidelines for PA and sedentary behavior for people with these selected chronic conditions are summarized in Table 3. As per the formal WHO Grading of Recommendations Assessment, Development and Evaluation process, ${ }^{24}$ these guidelines also considered the certainty and directness of evidence, the balance of benefits to harms, and if and when medical clearance and advice from health professionals is recommended.

\section{Discussion}

The population health impact of insufficient MVPA and the potential gains from even small population-wide increases in PA are well described and potentially substantial, ${ }^{45,46}$ supporting global efforts set out in the WHO GAPPA to achieve a $15 \%$ reduction in inactivity by $2030 .{ }^{13}$ These new WHO guidelines and supporting evidence affirm that adults living with chronic conditions who are or become more physically active can have reduced risk of premature mortality or disease progression and improved physical function and quality of life. Overall, the new WHO guidelines are complementary to existing clinical practice guidance, which states and reinforces the value, need, and benefits of PA in people living with chronic conditions. ${ }^{19-22}$ They also underscore that population-wide preventive efforts to increase PA and reduce sedentary behavior are relevant, safe, important, and beneficial not only for healthy adults but also for adults living with chronic conditions. These guidelines fill an important gap in global health policy and provide further support to global efforts under the United Nations Sustainable Development Goals Agenda: to achieve a one-third reduction in premature mortality from noncommunicable diseases through prevention and treatment, and promote mental health and wellbeing, by 2030 (goal 3.4). ${ }^{47}$

\section{T2D and Hypertension}

Physical activity, particularly MVPA, is already well-established within clinical guidance as a "first-line" treatment to help improve clinical risk factors and reduce disease progression in people with and at increased risk of T2D/CVD and hypertension. 19,21,22 Despite this well-established evidence base, PA intervention or referral is still far from being standard practice and remains comparatively underutilized. This may be due to a multitude of factors including: perceived low compliance or adverse health outcomes, lack of adequate training or inclusion in PA guidance in medical curricula, lack of incentives or prioritization, time constraints, and/or medicolegal responsibility. ${ }^{48}$ As such, these new recommendations reinforce the case for strengthening health systems and primary health care services and broader community-based programs to help prioritize and encourage integrated PA promotion at multiple levels. The WHO Global HEARTS Initiative ${ }^{49}$ and the Package of Essential Noncommunicable Disease Interventions ${ }^{50}$ are 2 examples of a prioritized set of cost-effective interventions, which incorporate PA and are designed for delivery in diverse settings. However, successful adoption and implementation will require appropriate training and adequate resourcing. ${ }^{51,52}$

\section{Cancer}

The importance of PA for improved patient-reported outcomes and premature mortality in cancer survivors has emerged only more recently ${ }^{33}$ but is now more formally recommended to be incorporated as part of regular clinical practice-for example the American College of Sports Medicine's Moving Through Cancer guidelines. ${ }^{20}$ The inclusion of PA guidelines for cancer survivors will improve awareness globally of the health benefits that exist with PA for people living with cancer. To date, there remains a general lack of knowledge of these benefits and, consequently, a lack of services and counselling to help guide cancer survivors to include regular PA as part of their lifestyles. However, this is a growing area and community-based programs, guidance, and research on their effectiveness are underway. ${ }^{53-55}$

\section{HIV}

New evidence syntheses included in this review highlight the important role of MVPA on physical function, mental health, and disease progression outcomes among adults living with HIV, along with a likely absence of adverse effects. Evidence from a large longitudinal cohort also suggests that benefits include improved cardiometabolic health and reduced odds of co-morbid conditions. ${ }^{56}$ Together, these new evidence reviews are important for the significant proportion of young people and aging adults living with HIV (approximately $~ 38$ million globally in 2019) alongside the direct complications and side effects of HIV antiretroviral therapies, which further increase the risk for adverse cardiometabolic sequelae. ${ }^{57}$ The new WHO guidelines will therefore generate greater awareness and understanding of the important benefits of PA for people living with HIV and should, ideally, stimulate new services, integration of counselling, and programs aimed at increasing PA levels and reducing sedentary behaviors.

\section{A Call to Action and Strengthening the Integration of PA into Primary Health Care}

As the global burden of chronic disease continues to grow, and despite repeated high-level calls for stronger action, ${ }^{4,5,47}$ global progress and implementation on the prevention and management of chronic diseases remains slow and uneven. ${ }^{4}$ Nevertheless, there is renewed emphasis on the critical role of primary health care for people living with chronic conditions. These guidelines confirm that there is sufficient evidence to integrate recommendations on PA and sedentary behavior into primary health care and, more broadly, increase opportunities to embed PA programs and policies into communitybased health services. However, securing the adoption and implementation within primary health care at scale will need leadership and greater prioritization. Indeed, a recent survey revealed that currently only one-third of countries (34\%; 65 of 194 Member states/countries) report having protocols on PA assessment in primary health care, ${ }^{58}$ and there are limited data on actual implementation.

These new global guidelines should serve as a potent catalyst for strengthening the implementation of more PA strategies and policies into primary health care and social services. Effective 
approaches are available and recommended in the GAPPA and the WHO ACTIVE Technical Package. ${ }^{13}$ For example, the WHO Global HEARTS Initiative, ${ }^{49}$ the Package of Essential Noncommunicable Disease Interventions, ${ }^{50}$ and Physical Activity Vital $S i g n^{59}$ all provide useful implementation guidance. Furthermore, there is a growing array of creative, pragmatic, and inspirational resources to help encourage and embed more condition-specific evidence-based PA advice into health care services. A few highquality examples include Exercise is Medicine ${ }^{\circledR}$ (American College of Sports Medicine), Motivate2Move, and Moving Medicine (United Kingdom), ${ }^{60}$ as well as online campaigns and open access programs (eg, We Are Undefeatable, United Kingdom) supporting those with chronic conditions to be more active.

Medical professionals have an important role to play. Specifically, they should routinely include a brief assessment of PA and sedentary behavior levels at every visit and advise/encourage patients to be more active (eg, by "Making every contact count" and by making PA a "Vital Sign" 59 though an "ask-assess-advise" model). ${ }^{13}$ Although more research is still needed on specific effectiveness and feasibility/accessibility of brief or very brief advice, ${ }^{61}$ these approaches help raise awareness among patients and other colleagues and provide important opportunities for discussion on the importance of PA. They also help in monitoring progress and identifying highly sedentary or physically inactive patients who may benefit the most from further follow-up, specific support, or intervention.

It should be noted that for those individuals with chronic conditions who are physical inactive and highly sedentary, and likely deconditioned or with functional limitations, recommendations to increase the volume of MVPA (ie, the second sedentary behavior recommendation; Table 3) may be more challenging. For these individuals, it may be more prudent and practical, at least in the first instance, to emphasize the first sedentary behavior recommendation of limiting the amount of time spent being sedentary and replacing it with some PA of any (feasible) intensity, before incrementally increasing the amount. Indeed, a safeguard against doing too much PA too soon is to "start slow and build up," taking into account individual capacity or functional limitations.

Importantly, systems and policies should be implemented that allow, empower, and incentivize medical professionals to provide or, ideally, refer inactive patients onto appropriate counselling, but also facilitate policies and programs to support patients in overcoming barriers to improve their activity levels. A multi-agency approach and better links with other health, behavioral, or exercise professionals will likely be required, and resources developed from the new WHO Global guidelines should be disseminated widely through various channels to improve reach. Training and intervention policies should be adapted to local contexts, cultures, and resource constraints, ensuring community and patient involvement and coordinated links with community-based resources and opportunities for PA and sedentary behavior change where appropriate. The guidelines also need to be well known and understood by appropriate influencers and the medical community (eg, taught and accepted in medical school curricula) and embedded within standard medical practice. Achieving this objective requires adequate investment in training programs for health, community, and social care providers in primary and secondary health care and social services. One example of this is the MovementForMovement community of practice (United Kingdom), which aims to support capacity building in the health care workforce by embedding and providing free evidence-based undergraduate health care teaching resources on PA. ${ }^{62-64}$

\section{Bridging Gaps to Better Integrate Primary Care and Population Health}

Strategies to prevent noncommunicable diseases and manage chronic conditions are often narrowly focused on clinical interventions or individual behavior change approaches alone. These approaches are important; however, it has been well-established that increasing PA and reducing sedentary behavior requires addressing a wider array of social, cultural, and environmental factors. ${ }^{65}$ This will require better integration and collaboration between primary care and population health-jurisdictions that, unfortunately, tend to mostly function in isolation. By linking clinical services to those of other sectors, such as community or population health (eg, through "community hubs" to provide more targeted PA, sedentary behavior, and other non-pharmacological support/services/approaches for people with chronic conditions), health care systems can enhance patient contact/follow-up and improve health outcomes, reduce duplication of services, and achieve better economies of scale by consolidating services across clinical care and population health programs. Improved integration would also allow clinical practices to match health care services and community support to specific chronic condition needs, thereby better targeting preventive efforts at higher-risk or more underserved populations and reducing overall health care burden. An emphasis on such collaborative approaches is already recommended in GAPPA. ${ }^{13}$

\section{Creating Health-Enhancing Environments}

As always, it also remains vital that actions beyond the health care setting are also adopted. ${ }^{13}$ Among the 20 recommended policies in GAPPA are calls for all countries to implement sustained national public education and awareness campaigns, as well as the creation of appropriate urban infrastructure and environments to support all people, including those with chronic conditions, to be more physically active. The WHO emphasizes that no single solution will be sufficient to change population levels of PA and sedentary behavior; rather, a whole of community or "systems-based" approach is needed to address the multiple determinants of inactivity, one that focuses on combining both upstream policy approaches and downstream individual-focused strategies. ${ }^{66}$ The new WHO guidelines call for the policy and program responses to be inclusive and address the needs, challenges, and contexts of those people living with chronic conditions.

\section{Future Research and Opportunities}

As the evidence base on the preventive health benefits of PA and sedentary behavior in adults living with chronic conditions is relatively sparse compared with otherwise healthy adults without a chronic condition, a number of important research gaps were identified by the GDG. ${ }^{67}$ The present review focused on 3 specific chronic conditions of high prevalence and public health burden and provided new evidence syntheses for HIV. This extends previous evidence reviews ${ }^{14}$ among people with 1 of several common chronic diseases or conditions, a number of which had insufficient evidence for several outcomes of interest (see Table D-3, pg. 86). There is, however, a clear need for more evidence (both from RCT and observational studies) on a broader range of chronic conditions and disabilities where PA and sedentary behavior would be expected to play a direct role (eg, specific cancers, obesity, chronic obstructive pulmonary disease/asthma, and musculoskeletal and brain/mood disorders). There is also a vital need for more evidence 
from low- and middle-income countries (including more evidence from the African region for HIV, as well as for other chronic conditions) and to address socioeconomic inequalities.

Ideally, such research would also incorporate data from device-based measures of PA and provide more detailed evidence on the different types, intensities, and optimum doses (or thresholds) of activity required to improve physical function and quality of life, as well as reduce the risk of comorbid conditions. Similarly, more direct evidence on the potential feasibility and health benefits of limiting sedentary behaviors and replacing them with more light-intensity activities (eg, standing and brief/intermittent activity breaks) could also be particularly pertinent for people living with chronic conditions, ${ }^{29}$ who are often more deconditioned, physically inactive, and sedentary, but could derive substantial benefits from simply moving more, more often. ${ }^{68,69}$ Finally, but equally important, will be the translation of observational and RCT evidence into clinical and public health practice and policy with the goal of achieving system embeddedness (ie, successful "scale-up") of interventions and programs as opposed to only short-term implementation or impact.

\section{Conclusions}

Demographic and economic forecasts show that noncommunicable diseases and the number of chronic health conditions per individual are increasing, placing undue burden on an already stretched primary health care system both in higher income and particularly low- and middle-income countries. The WHO 2020 Guidelines on Physical Activity and Sedentary Behavior provide new evidence and guidance on the types and amounts of PA and sedentary behavior to improve health, expanding beyond primary prevention into adults managing chronic conditions. Implementation of programs, practices, and policies to facilitate more PA and limit sedentary behavior could result in significant health and other co-benefits, as well as reduce the burden and cost of chronic disease to health care systems. It is hoped that these new guidelines will inform new system-wide policies, programs, and primary and community health care initiatives to help maximize benefits to global health, as well as prompt further research in this area. Clinical and public health professionals and policy makers should promote the new WHO global guidelines and develop and implement services and programmes to increase PA and limit sedentary behavior in adults living with chronic conditions.

\section{Acknowledgments}

Systematic reviews of evidence prepared for 2018 US PA Guidelines Advisory Committee Scientific Report to the Secretary of Health and Human Services were updated thanks to additional literature searches conducted by Kyle Sprow (National Cancer Institute, National Institutes of Health, Maryland, USA). Summaries of evidence and Grading of Recommendations Assessment, Development and Evaluation tables were prepared by Carrie Patnode and Michelle Henninger (The Kaiser Foundation Hospitals, Center for Health Research, Portland, Oregon, USA). The Public Health Agency of Canada and the Government of Norway provided financial support to update the WHO guidelines on PA and sedentary behavior. P.C.D. was supported by a National Health and Medical Research Council (NHMRC) of Australia Fellowship (number 1142685) and the UK Medical Research Council (MC_UU_12015/3). The authors declare that they have no competing interests.

\section{References}

1. Kyu HH, Abate D, Abate KH, et al. Global, regional, and national disability-adjusted life-years (DALYs) for 359 diseases and injuries and healthy life expectancy (HALE) for 195 countries and territories, 1990-2017: a systematic analysis for the Global Burden of Disease Study 2017. Lancet. 2018;392(10159):1859-1922. doi:10.1016/ S0140-6736(18)32335-3

2. Roth GA, Abate D, Abate KH, et al. Global, regional, and national age-sex-specific mortality for 282 causes of death in 195 countries and territories, 1980-2017: a systematic analysis for the Global Burden of Disease Study 2017. Lancet. 2018;392(10159):17361788. doi:10.1016/S0140-6736(18)32203-7

3. World Health Organization. Global Action Plan for the Prevention and Control of Noncommunicable Diseases 2013-2020. Geneva, Switzerland: World Health Organization; 2013. https://www.who. int/nmh/events/ncd_action_plan/en/.

4. World Health Organization. Time to Deliver: Report of the WHO Independent High-Level Commission on NCDs. Geneva, Switzerland: World Health Organization; 2018. https://www.who.int/ncds/ management/time-to-deliver/en/.

5. World Health Organization. Tackling NCDs: 'Best Buys' and Other Recommended Interventions for the Prevention and Control of Noncommunicable Diseases. Geneva, Switzerland: World Health Organization; 2017. https://apps.who.int/iris/handle/10665/259232.

6. GBD Obesity Collaborators, Afshin A, Forouzanfar MH, et al. Health effects of overweight and obesity in 195 countries over 25 years. $N$ Engl J Med. 2017;377(1):13-27.

7. Guthold R, Stevens GA, Riley LM, Bull FC. Worldwide trends in insufficient physical activity from 2001 to 2016: a pooled analysis of 358 population-based surveys with 1.9 million participants. Lancet Glob Health. 2018;6(10):e1077-e1086. doi:10.1016/S2214-109X(18)30357-7

8. Marengoni A, Angleman S, Melis R, et al. Aging with multimorbidity: a systematic review of the literature. Ageing Res Rev. 2011; 10(4):430-439. doi:10.1016/j.arr.2011.03.003

9. Hajat C, Stein E. The global burden of multiple chronic conditions: a narrative review. Prev Med Rep. 2018;12:284-293. doi:10.1016/j. pmedr.2018.10.008

10. Ding D, Lawson KD, Kolbe-Alexander TL, et al. The economic burden of physical inactivity: a global analysis of major noncommunicable diseases. Lancet. 2016;388(10051):1311-1324. doi:10.1016/S0140-6736(16)30383-X

11. Piercy KL, Troiano RP, Ballard RM, et al. The physical activity guidelines for Americans. JAMA. 2018;320(19):2020-2028. doi:10. 1001/jama.2018.14854

12. Marco H, Yerushalmi E, Phillips WD, et al. The Economic Benefits of a More Physically Active Population: An International Analysis. Santa Monica, CA: RAND Corporation; 2019.

13. World Health Organization. Global Action Plan on Physical Activity 2018-2030: More Active People for a Healthier World. Geneva, Switzerland: World Health Organization; 2018. https://www.who.int/ ncds/prevention/physical-activity/global-action-plan-2018-2030/en/.

14. US Department of Health and Human Services. 2018 Physical Activity Guidelines Advisory Committee Scientific Report. 2018. https://health.gov/paguidelines/second-edition/report.aspx.

15. Pedersen BK, Saltin B. Exercise as medicine-evidence for prescribing exercise as therapy in 26 different chronic diseases. Scand J Med Sci Sports. 2015;25(suppl):1-72. doi:10.1111/sms.12581

16. Swedish National Institute of Public Health. Physical Activity in the Prevention and Treatment of Disease. Sweden: Professional Associations for Physical Activity; 2010. https://www.folkhalsomyndigheten. 
se/contentassets/5de033c2c75a494a99cbba2407594c22/physicalactivity-prevention-treatment-disease-webb.pdf.

17. Vancampfort D, Koyanagi A, Ward PB, et al. Chronic physical conditions, multimorbidity and physical activity across 46 lowand middle-income countries. Int J Behav Nutr Phys Act. 2017; 14(1):6. doi:10.1186/s12966-017-0463-5

18. Vancampfort D, Stubbs B, Koyanagi A. Physical chronic conditions, multimorbidity and sedentary behavior amongst middle-aged and older adults in six low- and middle-income countries. Int J Behav Nutr Phys Act. 2017;14(1):147. doi:10.1186/s12966-017-0602-Z

19. Colberg SR, Sigal RJ, Yardley JE, et al. Physical activity/exercise and diabetes: a position statement of the American Diabetes Association. Diabetes Care. 2016;39(11):2065-2079. doi:10. 2337/dc16-1728

20. Schmitz KH, Campbell AM, Stuiver MM, et al. Exercise is medicine in oncology: engaging clinicians to help patients move through cancer. CA Cancer J Clin. 2019;69(6):468-484. doi:10.3322/caac.21579

21. Unger T, Borghi C, Charchar F, et al. 2020 International society of hypertension global hypertension practice guidelines. Hypertension. 2020; 75(6):1334-1357. doi:10.1161/HYPERTENSIONAHA.120.15026

22. Whelton PK, Carey RM, Aronow WS, et al. 2017 ACC/AHA/AAPA/ ABC/ACPM/AGS/APhA/ASH/ASPC/NMA/PCNA guideline for the prevention, detection, evaluation, and management of high blood pressure in adults: a report of the American College of Cardiology/ American Heart Association Task Force on clinical practice guidelines. Hypertension. 2018;71(6):e13-e115.

23. Bull F, Al-Ansari S, Biddle SJH, et al. World Health Organization 2020 guidelines on physical activity and sedentary behaviour. $\mathrm{Br} J$ Sports Med. 2020. In press. doi:10.1136/bjsports-2020-102955

24. World Health Organization. WHO Handbook for Guideline Development. 2nd ed. Geneva, Switzerland: World Health Organization; 2014. https://apps.who.int/iris/handle/10665/145714.

25. Piepoli MF, Hoes AW, Agewall S, et al. 2016 European guidelines on cardiovascular disease prevention in clinical practice: The Sixth Joint Task Force of the European Society of Cardiology and Other Societies on Cardiovascular Disease Prevention in Clinical Practice (constituted by representatives of 10 societies and by invited experts) developed with the special contribution of the European Association for Cardiovascular Prevention \& Rehabilitation (EACPR). Eur Heart J. 2016;37(29):2315-2381. doi:10.1093/eurheartj/ehw106

26. Anderson L, Thompson DR, Oldridge N, et al. Exercise-based cardiac rehabilitation for coronary heart disease. Cochrane Database Syst Rev. 2016;2016(1):CD001800.

27. Carty C, Van der Ploeg HP, Biddle SJH, et al. The first global physical activity and sedentary behaviour guidelines for people living with disability. J Phys Act Health. 2020. In press. doi:10.1123/jpah.20200629

28. Bull F, Willumsen J. World Health Organization Guidelines on Physical Activity and Sedentary Behaviour. Geneva, Switzerland: World Health Organization; 2020.

29. Dempsey PC, Biddle SJH, Buman MP, et al. New global guidelines on sedentary behaviour and health for adults: broadening the behavioural targets. Int J Behav Nutr Phys Act. 2020;17(1):151. doi:10. 1186/s12966-020-01044-0

30. Shea BJ, Reeves BC, Wells G, et al. AMSTAR 2: a critical appraisal tool for systematic reviews that include randomised or non-randomised studies of healthcare interventions, or both. BMJ. 2017; 358:j4008. doi:10.1136/bmj.j4008

31. Balshem H, Helfand M, Schunemann HJ, et al. GRADE guidelines: 3. Rating the quality of evidence. J Clin Epidemiol. 2011;64(4):401406. doi:10.1016/j.jclinepi.2010.07.015
32. Guyatt GH, Oxman AD, Vist GE, et al. GRADE: an emerging consensus on rating quality of evidence and strength of recommendations. BMJ. 2008;336(7650):924-926. doi:10.1136/bmj.39489.470347.AD

33. Friedenreich CM, Stone CR, Cheung WY, Hayes SC. Physical activity and mortality in cancer survivors: a systematic review and meta-analysis. JNCI Cancer Spectr. 2020;4(1):pkz080. doi:10.1093/ jncics/pkz080

34. Costa EC, Hay JL, Kehler DS, et al. Effects of high-intensity interval training versus moderate-intensity continuous training on blood pressure in adults with pre- to established hypertension: a systematic review and meta-analysis of randomized trials. Sports Med. 2018; 48(9):2127-2142. doi:10.1007/s40279-018-0944-y

35. Cao M, Quan M, Zhuang J. Effect of high-intensity interval training versus moderate-intensity continuous training on cardiorespiratory fitness in children and adolescents: a meta-analysis. Int J Environ Res Public Health. 2019;16(9):1533. doi:10.3390/ijerph16091533

36. Vatten LJ, Nilsen TI, Holmen J. Combined effect of blood pressure and physical activity on cardiovascular mortality. J Hypertens. 2006; 24(10):1939-1946. doi:10.1097/01.hjh.0000244941.49793.f9

37. Rossi A, Dikareva A, Bacon SL, Daskalopoulou SS. The impact of physical activity on mortality in patients with high blood pressure: a systematic review. J Hypertens. 2012;30(7):1277-1288. doi:10. 1097/HJH.0b013e3283544669

38. Hu G, Jousilahti P, Antikainen R, Tuomilehto J. Occupational, commuting, and leisure-time physical activity in relation to cardiovascular mortality among Finnish subjects with hypertension. Am J Hypertens. 2007;20(12):1242-1250. doi:10.1016/j.amjhyper.2007.07.015

39. Kodama S, Tanaka S, Heianza Y, et al. Association between physical activity and risk of all-cause mortality and cardiovascular disease in patients with diabetes: a meta-analysis. Diabetes Care. 2013;36(2): 471-479. doi:10.2337/dc12-0783

40. Sluik D, Buijsse B, Muckelbauer R, et al. Physical activity and mortality in individuals with diabetes mellitus: a prospective study and meta-analysis. Arch Intern Med. 2012;172(17):1285-1295. doi:10.1001/archinternmed.2012.3130

41. Sadarangani KP, Hamer M, Mindell JS, Coombs NA, Stamatakis E. Physical activity and risk of all-cause and cardiovascular disease mortality in diabetic adults from Great Britain: pooled analysis of 10 population-based cohorts. Diabetes Care. 2014;37(4):1016-1023. doi:10.2337/dc13-1816

42. Qiu S, Cai X, Sun Z, Zugel M, Steinacker JM, Schumann U. Aerobic interval training and cardiometabolic health in patients with type 2 diabetes: a meta-analysis. Front Physiol. 2017;8:957. doi:10.3389/ fphys.2017.00957

43. Liu Y, Ye W, Chen Q, Zhang Y, Kuo CH, Korivi M. Resistance exercise intensity is correlated with attenuation of $\mathrm{HbA1c}$ and insulin in patients with type 2 diabetes: a systematic review and metaanalysis. Int J Environ Res Public Health. 2019;16(1):140. doi:10. 3390/ijerph16010140

44. O'Brien KK, Tynan AM, Nixon SA, Glazier RH. Effectiveness of aerobic exercise for adults living with HIV: systematic review and meta-analysis using the Cochrane Collaboration protocol. BMC Infect Dis. 2016;16(1):182. doi:10.1186/s12879-016-1478-2

45. Strain T, Brage S, Sharp SJ, et al. Use of the prevented fraction for the population to determine deaths averted by existing prevalence of physical activity: a descriptive study. Lancet Glob Health. 2020;8(7): e920-e930. doi:10.1016/S2214-109X(20)30211-4

46. Lee IM, Shiroma EJ, Lobelo F, et al. Effect of physical inactivity on major non-communicable diseases worldwide: an analysis of burden of disease and life expectancy. Lancet. 2012;380(9838):219-229. doi:10.1016/S0140-6736(12)61031-9 
47. United Nations General Assembly. Transforming Our World: The 2030 Agenda for Sustainable Development. United Nations; 2015. https://sustainabledevelopment.un.org/post2015/transformingour world/publication.

48. Din NU, Moore GF, Murphy S, Wilkinson C, Williams NH. Health professionals' perspectives on exercise referral and physical activity promotion in primary care: findings from a process evaluation of the National Exercise Referral Scheme in Wales. Health Educ J. 2015;74(6):743-757. doi:10.1177/0017896914559785

49. World Health Organisation. Global Hearts Initiative: Working Together to Promote Cardiovascular Health. Geneva, Switzerland: World Health Organization; 2016. https://www.who.int/cardiovascular_ diseases/global-hearts/en/.

50. World Health Organization. Implementation Tools: Package of Essential Noncommunicable (PEN) Disease Interventions for Primary Health Care in Low-Resource Settings. Geneva, Switzerland: World Health Organization; 2013. https://www.who.int/nmh/ publications/essential_ncd_interventions_lr_settings.pdf.

51. Rogers HE, Akiteng AR, Mutungi G, Ettinger AS, Schwartz JI. Capacity of Ugandan public sector health facilities to prevent and control non-communicable diseases: an assessment based upon WHO-PEN standards. BMC Health Serv Res. 2018;18(1):606. doi:10.1186/s12913-018-3426-X

52. Aye LL, Tripathy JP, Maung Maung T, et al. Experiences from the pilot implementation of the package of essential non-communicable disease interventions (PEN) in Myanmar, 2017-18: a mixed methods study. PLoS One. 2020;15(2):e0229081. doi:10.1371/journal.pone. 0229081

53. Foster J, Worbey S, Chamberlin K, Horlock R, Marsh T. Integrating Physical Activity Into Cancer Care: Evidence and Guidance. London, UK: MacMillan Cancer Support; 2018.

54. Mina DS, Sabiston CM, Au D, et al. Connecting people with cancer to physical activity and exercise programs: a pathway to create accessibility and engagement. Curr Oncol. 2018;25(2):149-162. doi:10. $3747 /$ co. 25.3977

55. Sabiston CM, Fong AJ, O'Loughlin EK, Meterissian S. A mixedmethods evaluation of a community physical activity program for breast cancer survivors. J Transl Med. 2019;17(1):206. doi:10.1186/ s12967-019-1958-4

56. Willig AL, Webel AR, Westfall AO, et al. Physical activity trends and metabolic health outcomes in people living with HIV in the US, 2008-2015. Prog Cardiovasc Dis. 2020;63(2):170-177. doi:10. 1016/j.pcad.2020.02.005

57. Lagathu C, Bereziat V, Gorwood J, et al. Metabolic complications affecting adipose tissue, lipid and glucose metabolism associated with HIV antiretroviral treatment. Expert Opin Drug Saf. 2019;18(9):829840. doi:10.1080/14740338.2019.1644317

58. World Health Organization. Assessing National Capacity for the Prevention and Control of Noncommunicable Diseases: Report of the 2019
Global Survey. Geneva, Switzerland: World Health Organization; 2020. https://www.who.int/publications/i/item/ncd-ccs-2019.

59. Sallis RE, Matuszak JM, Baggish AL, et al. Call to action on making physical activity assessment and prescription a medical standard of care. Curr Sports Med Rep. 2016;15(3):207-214. doi:10.1249/JSR. 0000000000000249

60. Brannan M, Bernardotto M, Clarke N, Varney J. Moving healthcare professionals - a whole system approach to embed physical activity in clinical practice. BMC Med Educ. 2019;19(1):84. doi:10.1186/ s12909-019-1517-y

61. Lamming L, Pears S, Mason D, et al. What do we know about brief interventions for physical activity that could be delivered in primary care consultations? A systematic review of reviews. Prev Med. 2017;99:152-163. doi:10.1016/j.ypmed.2017.02.017

62. Gates AB, Swainson MG, Isba R, Wheatley RG, Curtis FA. Movement for movement: a practical insight into embedding physical activity into the undergraduate medical curriculum exemplified by Lancaster Medical School. Br J Sports Med. 2019;53(10):609-610. doi:10.1136/bjsports-2018-100243

63. Gates AB, Kerry R, Moffatt F, et al. Movement for movement: exercise as everybody's business? Br J Sports Med. 2017;51(10): 767-768. doi:10.1136/bjsports-2016-096857

64. Gates AB, Swainson MG, Moffatt F, Kerry R, Metsios GS, Ritchie I. Undergraduate examination and assessment of knowledge and skills is crucial in capacity planning for the future healthcare workforce in physical activity interventions. Br J Sports Med. 2020;54(17):10151016. doi:10.1136/bjsports-2019-101646

65. Sallis JF, Cervero RB, Ascher W, Henderson KA, Kraft MK, Kerr J. An ecological approach to creating active living communities. Annu Rev Public Health. 2006;27(1):297-322. doi:10.1146/annurev. publhealth.27.021405.102100

66. Global Advocacy for Physical Activity (GAPA) the Advocacy Council of the International Society for Physical Activity and Health (ISPAH). NCD prevention: investments that work for physical activity. Br J Sports Med. 2012;46(10):709-712. doi:10.1136/bjsm. 2012.091485

67. DiPietro L, Al-Ansari S, Biddle SJH, et al. Advancing the global physical activity agenda: recommendations for future research by the 2020 WHO physical activity and sedentary behavior guidelines development group. Int J Behav Nutr Phys Act. 2020;17(1):143. doi:0.1186/s12966-020-01042-2

68. Dempsey PC, Larsen RN, Dunstan DW, Owen N, Kingwell BA. Sitting less and moving more: implications for hypertension. Hypertension. 2018;72(5):1037-1046. doi:10.1161/HYPERTEN SIONAHA.118.11190

69. Dempsey PC, Owen N, Yates TE, Kingwell BA, Dunstan DW. Sitting less and moving more: improved glycaemic control for type 2 diabetes prevention and management. Curr Diab Rep. 2016;16(11): 114. doi:10.1007/s11892-016-0797-4 